\title{
The Impact of Tides on the Capillary Transition Zone
}

\author{
Stefan Iglauer • Ann Muggeridge
}

Received: 29 February 2012 / Accepted: 15 December 2012 / Published online: 10 January 2013

(C) The Author(s) 2013. This article is published with open access at Springerlink.com

\begin{abstract}
The capillary transition zone, also known as the capillary fringe, is a zone where water saturations decrease with height above the water table/oil-water contact as a result of capillary action. In some oil reservoirs, this zone may contain a significant proportion of the oil in place. In groundwater assessments, the capillary fringe can profoundly affect contaminant transport. In this study, we investigated the influence of a tidally induced, semidiurnal, change in water table depth on the water saturation distribution in the capillary fringe/transition zone. The investigation used a mixture of laboratory experiments, in which the change in saturation with depth was monitored over a period of 90 days, and numerical simulation. We show that tidal changes in water table depth can significantly alter the vertical water saturation profile from what would be predicted using capillary-gravity equilibrium and the drainage or imbibition capillary pressure curves.
\end{abstract}

Keywords Capillary fringe $\cdot$ Transition zone $\cdot$ Hydrocarbon $\cdot$ Groundwater $\cdot$ Tides

\section{List of symbols}

$\begin{array}{ll}a & \text { Tortuosity factor in Archie's law } \\ d & \text { Depth of water table in absence of tidal forcing (m) } \\ K & \text { Hydraulic conductivity }\left(\mathrm{m} \mathrm{s}^{-1}\right) \\ m & \text { Cementation exponent } \\ n & \text { Saturation exponent in Archie's law } \\ S_{\mathrm{w}} & \text { Water saturation (fraction) } \\ r_{r m w} & \text { Resistivity of brine (ohm m) } \\ r & \text { Resistivity of sand pack (ohm m) }\end{array}$

S. Iglauer

Department of Petroleum Engineering, Curtin University, Perth, WA, Australia

A. Muggeridge $(\bowtie)$

Department of Earth Science and Engineering, Imperial College, London SW7 2AZ, UK

e-mail: a.muggeridge@imperial.ac.uk 


$\begin{array}{ll}R_{0} & \text { Resistance between electrodes when } S_{\mathrm{W}}=1(\mathrm{ohm}) \\ R & \text { Resistance between electrodes }(\mathrm{ohm}) \\ t & \text { Time (s) }\end{array}$

\section{Greek symbols}

$\begin{array}{ll}\phi & \text { Porosity (fraction) } \\ \omega & \text { Angular frequency of tidal forcing }\left(\mathrm{s}^{-1}\right)\end{array}$

\section{Subscripts}

$\begin{array}{ll}d & \text { Dynamic effective } \\ w & \text { Water }\end{array}$

\section{Introduction}

Many oil reservoirs contain a significant transition zone, where oil and water saturations change with height above the oil-water contact (e.g. Parker and Rudd 2000; Fanchi et al. 2002; Jackson et al. 2005) as a result of capillary gravity equilibrium (Dake 1983). This zone can contain significant volumes of oil, particularly in lower permeability formations. It is therefore important to be able to predict the vertical profile of saturation versus depth when estimating initial volumes of oil in place and when designing oil recovery schemes. This zone is also seen above the water table, where it is termed the capillary fringe. It is important to understand the water-air distribution in this fringe in order to better describe contaminant transport in groundwater (e.g. Bunn et al. 2010) as well as changing water levels in aquifers.

In theory, the water saturation distribution with depth can be predicted if the capillary pressure curve for the formation and the density difference between the fluids are known. In practice, however, the saturation versus depth inferred from log data is often very different from that based on capillary pressure curves measured in the laboratory (Fanchi et al. 2002; Masmaleh et al. 2007). Often this difference is ascribed to formation heterogeneity and changes in wettability with depth and saturation (Fanchi et al. 2002; Jackson et al. 2005; Masmaleh et al. 2007; Bunn et al. 2010).

There are a number of studies in the groundwater literature suggesting that cyclic changes in water table depth may result in a higher than expected average water saturation above the water table (Lehmann et al. 1998; Li et al. 2000; Nielsen and Perrochet 2000; Ataie-Ashtiani et al. 2001; Stauffer and Kinzelbach 2001; Werner and Lockington 2003; Cartwright et al. 2005, 2009; Wu and Zhuang 2010). The majority of these investigated harmonic changes in water table depth with frequencies corresponding to those of sea waves arriving at a beach (Lehmann et al. 1998; Li et al. 2000; Nielsen and Perrochet 2000; Werner and Lockington 2003; Cartwright et al. 2005, 2009). Ataie-Ashtiani et al. (2001) and Wu and Zhuang (2010) considered the effect of tidal forcing on the water table although their focus was on determining the changing effects of the tides on the water table with distance from the coast. Neeper (2001) showed that tidally induced oscillations in an aquifer may also increase vertical diffusivity over that which would occur if the pore water were static.

Hydrocarbon reservoirs are typically found at greater depths and are unlikely to be influenced directly by waves, but they may nonetheless be affected by tidally induced changes in pressure (e.g. Langaas et al. 2006). Tidal effects on pressure and water table depth have been observed in bore-holes since the middle of the nineteenth century (Hanson and Owen 1982) 
and were first reported to have been observed in pressure data from petroleum reservoirs by Kuruana (1976). In the North Sea, formation pressure variations of between 700 and 3,500 Pa have been reported (Dean et al. 1994). Over the years, a number of authors have proposed using measurements of the tidal pressure response in aquifers and hydrocarbon reservoirs to infer their specific storage, porosity and compressibility (e.g. Bredehoeft 1967; Dean et al. 1994; Chang and Firoozabadi 2000).

In this paper, we investigate how the vertical saturation profile in the transition zone may be affected by tidally influenced changes in water table depth. An air-water transition zone was established in three 1-m high, sand filled columns. A cosinusoidally varying change in airwater contact was then applied to the columns for 90 days. The frequency of this change corresponded to that of the M2 semidiurnal, lunar tide (Butikov 2002). The water saturation versus depth was monitored continuously in all the columns throughout the 90 days. A 1D numerical model was built to predict experimental observations and to investigate the effect of tides on capillary-gravity equilibrium and saturation in the transition zone over a longer time-scale.

\section{Experimental Methods and Materials}

In order to investigate the effect of tidally induced pressure changes on the transition zone/capillary fringe, it was necessary to design a model system that would mimic reservoir properties and flows on laboratory length and time-scales. For this investigation, we decided to approximate the key features of the reservoir rather than exactly reproduce reservoir properties and conditions. For example, real transition zones in oil-water systems may have a vertical thickness of $>10 \mathrm{~m}$ (Fanchi et al. 2002; Jackson et al. 2005) whereas in the laboratory, it is impractical to pack a column of this height. In the reservoir, oil-water gravity drainage will occur over millions of years after reservoir filling whereas the objective of this investigation was to observe the interaction of gravity drainage and tidal pressure variations over a period of several months. It was also decided to maximize the possible effects of tidally induced changes in pressure on the transition zones.

These objectives were achieved by using a high permeability porous medium (unconsolidated sand rather than reservoir rock) and fluids with a high density difference (air and water rather than oil and water) to reduce the height of the transition zone and increase the rate of gravity drainage. The synthetic tidally induced pressure changes were then kept similar to those observed by Dean et al. (1994).

\subsection{Fluid Properties}

Air and brine were chosen as the two fluids used in these experiments. Their density difference is sufficiently large that a small $(\sim 1 \mathrm{~m})$, but nonetheless measurable transition zone could be produced in the laboratory. Brine was chosen rather than water so that electrical resistivity would be more sensitive to changes in saturation with location in the packs and with time. The brine was composed of $5 \mathrm{wt} \% \mathrm{NaCl}$ and $1 \mathrm{wt} \% \mathrm{KCl}$. The physical properties of the brine and air are given in Table 1.

\subsection{Porous Medium Properties}

Ottawa 42 sand (U.S. silica) was chosen as the porous medium for this study. The sand was strongly water-wet being composed of more than $99.8 \mathrm{wt} \%$ quartz. The grain size distribution was determined by sieving for $80 \mathrm{~min}$ on an electrical shaker using standard British meshed 
Table 1 Air and brine properties at ambient conditions of 0.101 M.Pa and 293.15 K unless stated

\begin{tabular}{ll}
\hline Property & Value \\
\hline Air viscosity & $1.88 \times 10^{-5} \mathrm{~Pa} \mathrm{~s}^{\mathrm{a}}$ \\
Brine viscosity & $1.085 \times 10^{-3} \mathrm{~Pa} \mathrm{~s}^{\mathrm{b}}$ \\
Air density & $1.2 \mathrm{~kg} \mathrm{~m}^{-3 \mathrm{c}}$ \\
Brine density & $1,040 \mathrm{~kg} \mathrm{~m}^{-3 \mathrm{c}}$ \\
Brine electrical resistivity & $0.127 \mathrm{Ohm} \mathrm{m}^{\mathrm{d}}$ \\
Air electrical conductivity & $<0.5 \mu \mathrm{S} \mathrm{cm}^{-1 \mathrm{e}}$ \\
Quartz resistivity & $7.5 \times 10^{17} \mathrm{Ohm} \mathrm{m}^{\mathrm{f}}$ \\
Air-brine interfacial tension & $72 \mathrm{mN} \mathrm{m}^{-1 \mathrm{a}}$ \\
\hline
\end{tabular}

${ }^{\text {a }}$ Reference temperature 298.15 K (Lide 2007)

b $5 \mathrm{wt} \% \mathrm{NaCl}$ brine (no $\mathrm{KCl}$ present) (Lide 2007)

${ }^{c}$ Measured in-house with an Anton Paar DMA 48

d Measured with Metrohm 712 Conductometer

e Not detectable with Metrohm 712 Conductometer

f Serway (1998)

Table 2 The porous medium properties measured using a secondary pack similar to those used for the gravity drainage and tidally influenced experiments

\begin{tabular}{ll}
\hline Property & Value \\
\hline Porosity & $35.4 \pm 1.3 \%$ \\
Permeability & $42.0 \pm 4.0 \mathrm{D}$ \\
F42 mean grain size & $290 \pm 82 \mathrm{~m}$ \\
\hline
\end{tabular}

sieves. Porosity, permeability and air-brine capillary pressure curves (drainage and imbibition) were measured using a small secondary pack with a similar geometry and inlet/outlet configuration to the main columns used for the experiments. The capillary pressure curves were determined by the porous plate method (Dullien 1992). The mean grain size, porosity and permeability values are given in Table 2 . The capillary pressure curves are shown in Fig. 1. These data are consistent with those presented by Camps-Roach et al. (2010) for F32/F50 Ottawa sand and by O'Carroll et al. (2005) for F35/F50 Ottawa sand.

\subsection{Preparation of the Packed Columns}

Three, identical, 1-m long, molded, poly-methyl-methacrylate columns with an internal diameter of $10 \mathrm{~cm}$ were used for the main set of experiments. The height of $1 \mathrm{~m}$ was selected to be slightly larger than the height of the air-water capillary transition zone that would be observed in Ottawa 42 sand.

The change in water saturation over time at nine different depths was measured indirectly by monitoring the resistance across a pair of electrodes at the chosen depths. This method has previously been used by Wakeham and Vince (1986) and Iglauer and Muggeridge (2012) among others. It has the advantage over most other methods in that it can be measured continuously over time without disturbing the pack. Its limitations when there is a nonuniform front are discussed in more detail by Aggelopoulos et al. (2005) and in cases when there are also pressure changes are discussed by (for example) Soner et al. (1996). We felt these limitations would be minimal in our experiments as the flow would be gravity dominated 


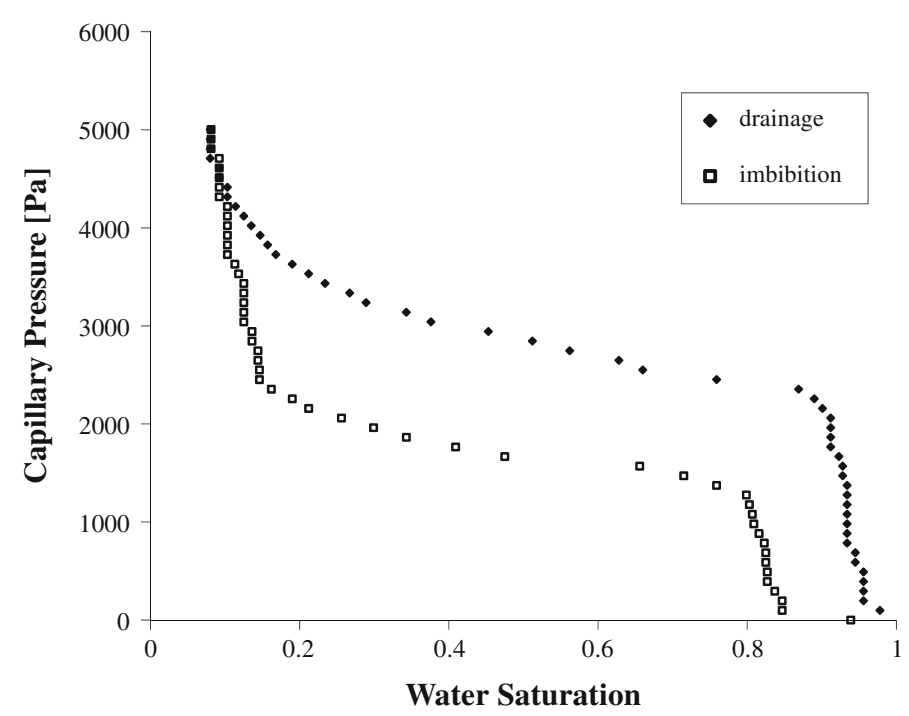

Fig. 1 The drainage and imbibition capillary pressure curves measured for the F42 Ottawa sand

so the air-water front was likely to be horizontal at all times and the changes in pressure were limited to $\sim 4,000 \mathrm{~Pa}$.

Thus each column was fitted with nine, non-corrosive stainless steel (Hastelloy) electrode pairs, placed at $10 \mathrm{~cm}$ intervals up the column, on opposite sides, to measure the resistivity at different depths. Figure 2 illustrates the electrical circuit used. A $60 \mathrm{~Hz}, 8 \mathrm{~V}$ peak amplitude AC power supply (Farnell Sine Square Oscillator LFM4) was connected across each electrode pair in sequence. The voltage drop across each electrode pair was recorded as a function of time together with the electrical current calculated from the voltage drop measured across a 20,000 Ohm $\pm 0.1 \%$ precision resistor using a Daqlab 2005 (with DBK15 and DBK80 cards, Adeptscience) data logging system.

Before packing, a circular piece of plastic mesh and two circular pieces of filter paper (VWR Int. Filter Papers 415) were placed in the bottom of each column to prevent sand leakage during the experiments. The column was then partially filled with water and sand was poured continuously into each column until it was full of sand, taking care to ensure that the water level was always above that of the sand in order to minimize the amount of air trapped in the pack. Once a column was completely filled with sand and brine, each pack was consolidated by tapping (vibrating) and then more sand was added until the column was completely filled again. Finally, a $0.5-\mathrm{m}$ plastic tube was connected to each column inlet, and the end of the tube was sealed with Parafilm ${ }^{\mathrm{TM}}$ to minimize water evaporation. The pore volume and porosity of each column was determined by mass balance. The values are given in Table 3.

\subsection{Experimental Procedure}

Initially all three columns were full of brine. The resistance across each pair of electrodes was measured for $24 \mathrm{~h}$. After $24 \mathrm{~h}$, the bottom outlets were opened at the same time as the Parafilm $^{\mathrm{TM}}$ sealing around the top inlets to each column was pierced. This allowed air to enter each column from the top and the brine to drain freely from the bottom of each column. 
Fig. 2 Sketch of one of the packed columns showing the inlet and outlet drain holes and the nine electrode pairs. The electrodes were switched in series and the voltage drop $\left(V_{1}, V_{2}, \ldots, V_{18}\right)$ across each sandpack section was measured versus time. $V_{\mathrm{S}}$ is the power supply, and $R$ a precision resistor

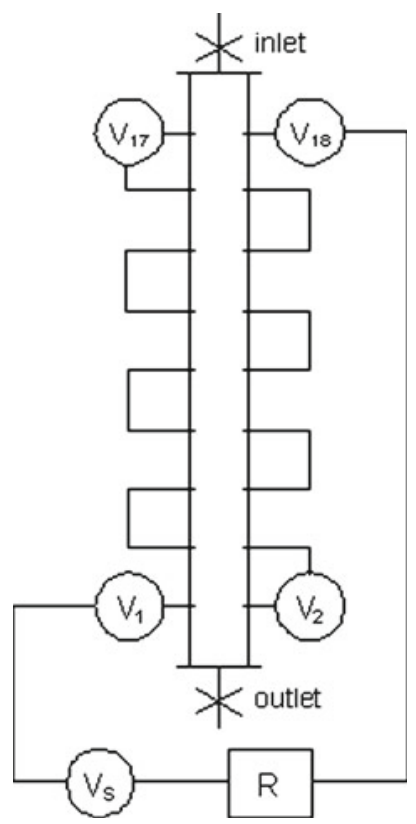

Table 3 The pore volume and porosity of each of the three columns when packed with Ottawa sand

\begin{tabular}{llc}
\hline Column & Pore volume $(\mathrm{ml})$ & Porosity \\
\hline 1 & 3068 & 0.42 \\
2 & 2688 & 0.36 \\
3 & 2972 & 0.40 \\
Average & 2910 & 0.39 \\
\hline
\end{tabular}

Columns 1 and 3 have very similar properties, while column 2 appears to be slightly more densely packed than the others

The brine was allowed to drain from the columns for a period of $24 \mathrm{~h}$ during which time the volume of brine produced from each column was collected and measured using a mass balance (see Iglauer and Muggeridge 2012). We continued to monitor the resistance across each pair of electrodes.

After $24 \mathrm{~h}$ of primary gravity drainage the inlet and outlet to each column was closed. The bottom outlets were then connected simultaneously to a brine reservoir mounted on a platform attached to a linear slide/rapid guide screw/stepper motor system (Reliance Precision Cool Muscle 23). The stepper motor had been programmed with CoolWorks Lite4.1.4 software to move the platform up and down with the height changing sinusoidally in time $(50,000 \mathrm{steps} /$ period). The period of this motion was $12 \mathrm{~h}$ and $20 \mathrm{~min}$ (the period of the lunar M2 semi-diurnal tide) and its amplitude was $\pm 20 \mathrm{~cm}$ (corresponding to a pressure change of 4,000 Pa, comparable with tidal pressure changes noted by Dean et al. 1994). The accuracy of this stepper motor system was $\pm 0.1 \%$.

The film sealing the top of each column was then pierced and the valve to the bottom of each column was opened allowing the brine from the reservoir to enter each column and establish an initial air-water contact $15 \mathrm{~cm}$ from the bottom of each column. The stepper motor was then 
turned on so that the reservoir was continuously moved up and down for $\sim 90$ days. This raised and lowered the air-water contact in each column with the same frequency and amplitude. The data logger continued to record resistance across each pair of electrodes throughout this time. The period of 90 days was chosen based on the gravity drainage investigations of Iglauer and Muggeridge (2012). They found that the time dependence of gravity drainage in these columns (in the absence of tidal forcing) could be modeled approximately by an exponential decay with a time constant of between 3 and 21 days. On this basis, the change in water saturation due to gravity drainage after 90 days would be below the resolution of the resistance measurements unless there was an ongoing impact of tidal forcing.

\subsection{Data Analysis}

We converted the resistances measured across each electrode over time using an equation derived from Archie's law (Archie 1942). Archie's law relates resistivity to water saturation and porosity through the relationship:

$$
r=\frac{a}{\phi^{m}} \frac{r_{\mathrm{w}}}{S_{\mathrm{w}}{ }^{n}}
$$

where $a$ is the tortuosity factor, $m$ is the cementation exponent, $r_{\mathrm{w}}$ is the resistivity of the brine, and $n$ is the saturation exponent.

The measured resistance depended upon the distance between the electrodes, the shape factor describing the geometry of the path followed by the current passing between the electrodes and the porosity and cementation of the porous medium between the electrodes as well as the water saturation. Assuming that the resistance of the water filled sand was much higher than the other contributions to measured resistance and that only water saturation changed over time, we can estimate the water saturation directly from the measured resistance through the relationship:

$$
S_{\mathrm{W}}=\left(\frac{R_{0}}{R(t)}\right)^{\frac{1}{n}},
$$

where $R(t)$ is the resistance measured across any electrode pair at a given time $t$ during drainage, and $R_{0}$ is the resistance measured across the same electrode pair before drainage begins, when the column is fully brine saturated. Following Jackson et al. (1978), we took $n=1.4$ (the value they obtained experimentally for clean, unconsolidated quartz sand).

\section{Numerical Simulation}

Numerical simulation was performed to inform our physical understanding of these experiments. The advantage of the simulator is that it directly predicts water saturation over time whereas our experiments measured resistance which is related to saturation but, as can be seen by examination of Eq. (1), is also dependent on porosity. Comparison of the experimental results with predictions from simulations gave us confidence that the observed changes were due primarily to changes in water saturation rather than porosity.

These investigations were performed using a commercial oil reservoir simulator, Eclipse 100 (produced by Schlumberger GeoQuest). This models Darcy flow in porous media including gravity and capillary pressure effects. 


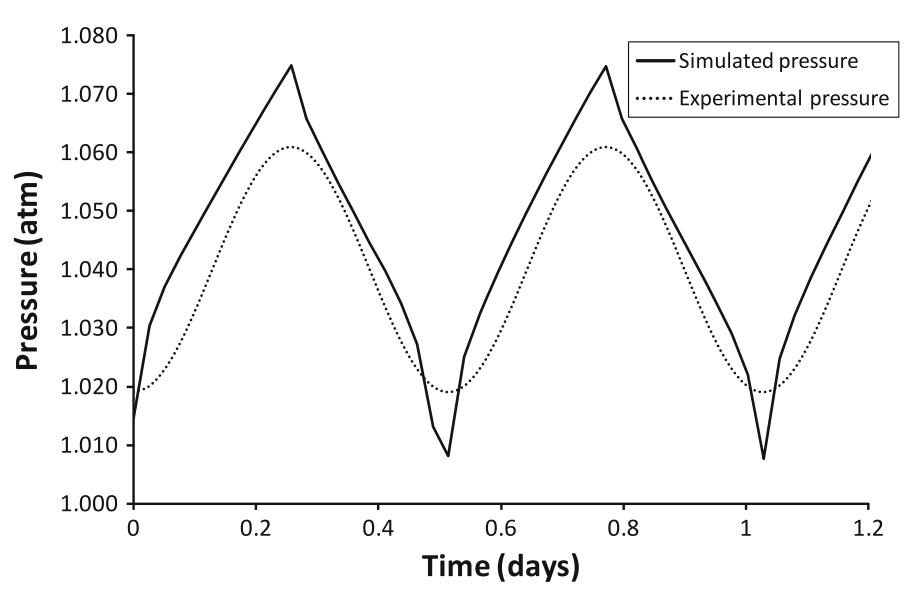

Fig. 3 The change in pressure applied to the bottom of the pack during the experimental compared with that seen in the numerical simulations. The sinusoidally changing pressure applied to the laboratory experiments became a saw-tooth change in the simulation because of the use of constant rate injection and production

A 1 D $1 \times 1 \times 50$ grid was used for the simulations. This grid size was selected on the basis of grid refinement studies. Further refinement in the vertical direction did not significantly change the predicted saturation distribution over time.

The simulator calculated the initial vertical water saturation distribution in the model of the experimental columns from the specified free water level and the drainage capillary pressure data. The experimental boundary conditions were approximated using a water injection well and a water production well completed in the bottom grid block of the model and an air injection and production well completed in the top grid block of the model. The water injection and production wells represented the tube connected to the water reservoir in the experiments and the air injection and production wells replicated the fact that the top of the columns were open to the air via a plastic tube. All wells were controlled by rate. We modeled the periodic changes in water oil contact by alternately injecting water at $176 \mathrm{~cm}^{3} \mathrm{~h}^{-1}$ for $6 \mathrm{~h}$ and $10 \mathrm{~min}$ with the air production well producing at a rate of $176 \mathrm{~cm}^{3} \mathrm{~h}^{-1}$ and the water production well shut in and then producing water at a rate of $176 \mathrm{~cm}^{3} \mathrm{~h}^{-1}$ for $6 \mathrm{~h} 10$ min with the air injection well open and injected air at a rate of $176 \mathrm{~cm}^{3} \mathrm{~h}^{-1}$ and the water injection well shut in. This resulted in a saw-tooth change in pressure in the simulations rather than the sinusoidal change in pressure applied to the experiment over time (Fig. 3). We used this approach because it was not possible to continuously change bottom hole pressure in the wells in the simulator to mimic the synthetic tidal variations. This method has been previously used by Ivanov and Araujo (2006).

Laboratory measurements of properties were used as input to the simulation where available. Although the simulator is able to model both relative permeability and capillary pressure hysteresis using the method of Killough (1976), we only chose to include capillary pressure hysteresis effects. This was because we had no data for imbibition relative permeability. We felt this was a reasonable approximation given that flow was dominated by gravity and capillary effects. The drainage relative permeability curves were obtained by history matching the first hour of primary gravity drainage. The Wyllie and Rose (1950) correlation was used for both the water and air relative permeability curves. Figure 4 shows the relative permeability curves used. 


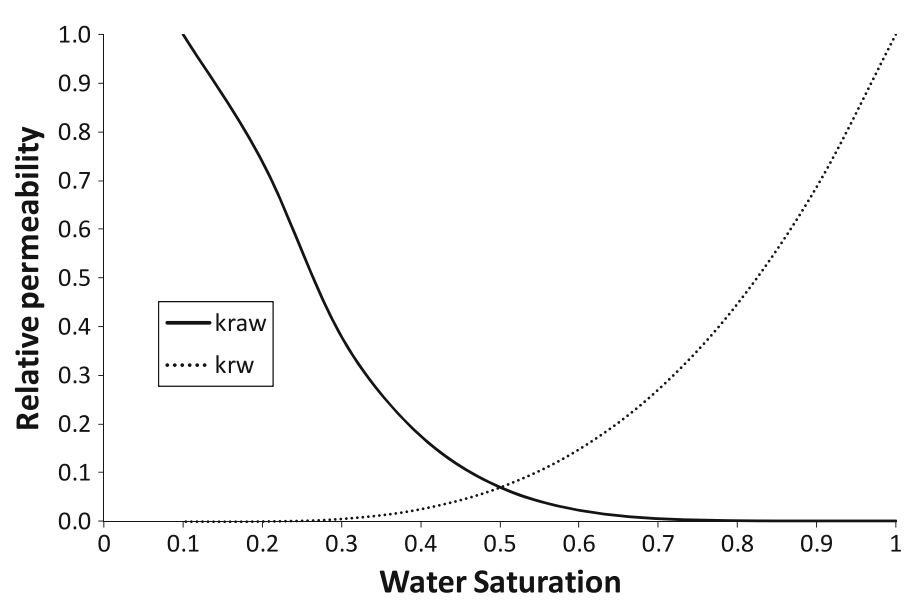

Fig. 4 The relative permeability curves obtained by history matching primary gravity drainage. These curves were used in all simulations described in this paper

\section{Results}

Figure 5 shows the initial water saturation versus depth seen in each column after $24 \mathrm{~h}$ of gravity drainage and the subsequent injection of water at the base to create an initial free water level at a depth of $85 \mathrm{~cm}$. At this point tidal forcing had not begun. The initial conditions in the simulation are also shown. Overall, there is good agreement between experiments and simulation although the experimental water saturations are systematically higher than those seen in the simulation. It can be seen that there is a higher water saturation at the top of the experimental columns in addition to the high water saturation at the bottom corresponding to the free water level. This is a manifestation of the capillary end effect that is often seen in corefloods (see Huang and Honarpour 1996 for example). Apart from this end effect the observed water saturation was approximately constant at around 0.25 between 20 and $50 \mathrm{~cm}$ below the top of each column and then increased gradually (as would be expected from capillary-gravity equilibrium) towards the free water level. The fluctuations in saturation seen in the experiments are real and are probably due to minor heterogeneities in packing leading to heterogeneities in the water saturation. Similar fluctuations in water saturation were seen by Sahni (1998) and reported in Di Donato et al. (2006) when monitoring air-water gravity drainage using CT scanning to measure water saturation versus depth.

It should be noted that the water saturations estimated from resistance occasionally become greater than 1. This is first seen here in Fig. 5 at $90 \mathrm{~cm}$ depth in columns 1 and 2. This is probably due to an increase in porosity in these packs during water injection which is not accounted for in Eq. (2). The packs were unconsolidated sand, and thus it is likely the sand grains moved apart when pressure in the packs was increased during injection. This increased the pack porosity, especially close to the point of injection. From Eq. (1), we see that an increase in porosity will reduce resistivity. As we have normalized our resistance measurements to resistance measured when the columns were $100 \%$ water saturated, before the initiation of tidal forcing, any subsequent small increases in porosity will reduce the measured resistance slightly and appear as water saturations greater than 1 . For this reason, we have labeled our graphs 'water saturation' to highlight that these data include other effects. This change in resistivity with pressure is seen to a much greater extent when performing core tests at reservoir conditions to calibrate resistivity logs (e.g. Soner et al. 1996). 
Fig. 5 Estimated water saturation versus depth observed in the three columns after $24 \mathrm{~h}$ primary drainage. These were measured after the columns were connected to the water reservoir and an initial air-water contact was established but before the tidal forcing was initiated. The initial water saturation profile used in the simulator is also shown with the simulated water saturations having been averaged over $\pm 5 \mathrm{~cm}$ the depth of each detector

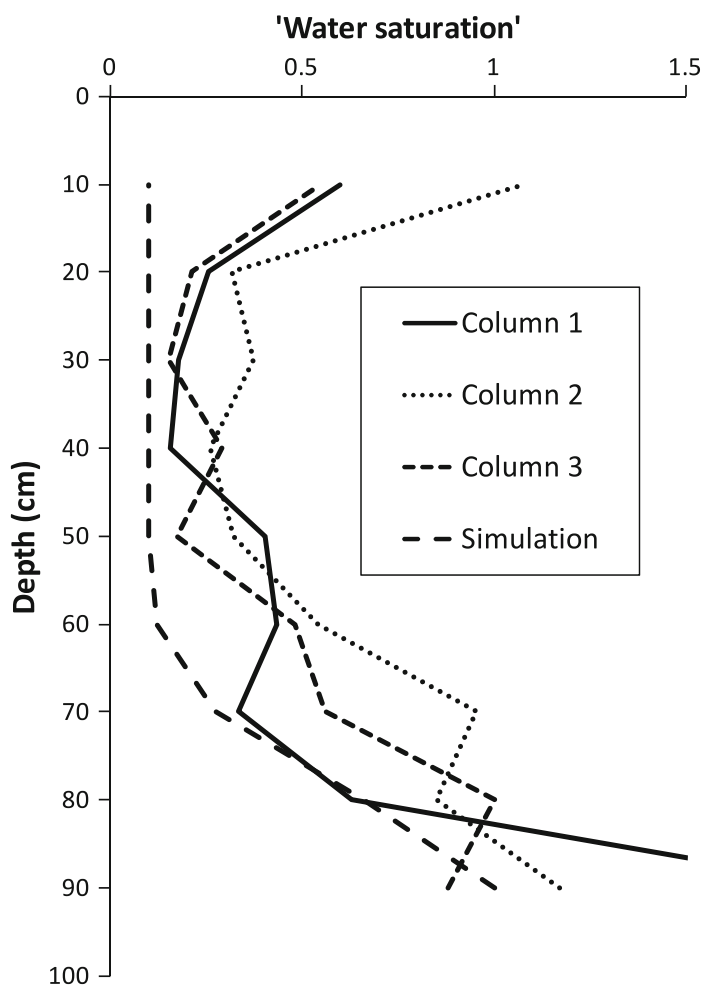

Figure 6 compares the 'saturation' measured in column 3 as a function of time after the synthetic tidal forcing was applied with that obtained from numerical simulation. The other two columns showed a similar response but with a smaller amplitude. It can be seen that 'saturation' changes periodically with time at all levels in the column although the changes in the bottom $20 \mathrm{~cm}$ and the top $20 \mathrm{~cm}$ of the columns were very small. The maximum change in 'water saturation' with time is observed between 30 and $50 \mathrm{~cm}$. These depths correspond to the depths over which the capillary transition zone formed and was affected when the free water level was moved by the 'tidally influenced' water injection.

It is interesting to see that 'saturation' changes in the columns were not always purely sinusoidal in response to the sinusoidally changing pressure. At depths of $70-80 \mathrm{~cm}$ in the column, the 'saturation' changes take the form of a sinusoid truncated at the maximum water saturation. At depths of $20-30 \mathrm{~cm}$, the saturation response is more of an asymmetric sawtooth: the 'water saturation' increased rapidly in phase with the change in depth of the free water level but then decayed away more slowly, presumably because the time-scale for gravity drainage in these packs is rather greater than the 12-h period of the tidal changes in pressure (see Iglauer and Muggeridge 2012). This asymmetric shape in the saturation change at these depths was seen more clearly in the simulation results, although it should be remembered that the tidal forcing in the simulations took the form of a saw-tooth rather than a sinusoid. Wu and Zhuang (2010) also observed an asymmetry in the height of the water table in their experiments using sand although in their case they saw the opposite effect: the water table height changed more slowly during the rising tide and more quickly during a falling tide. We 

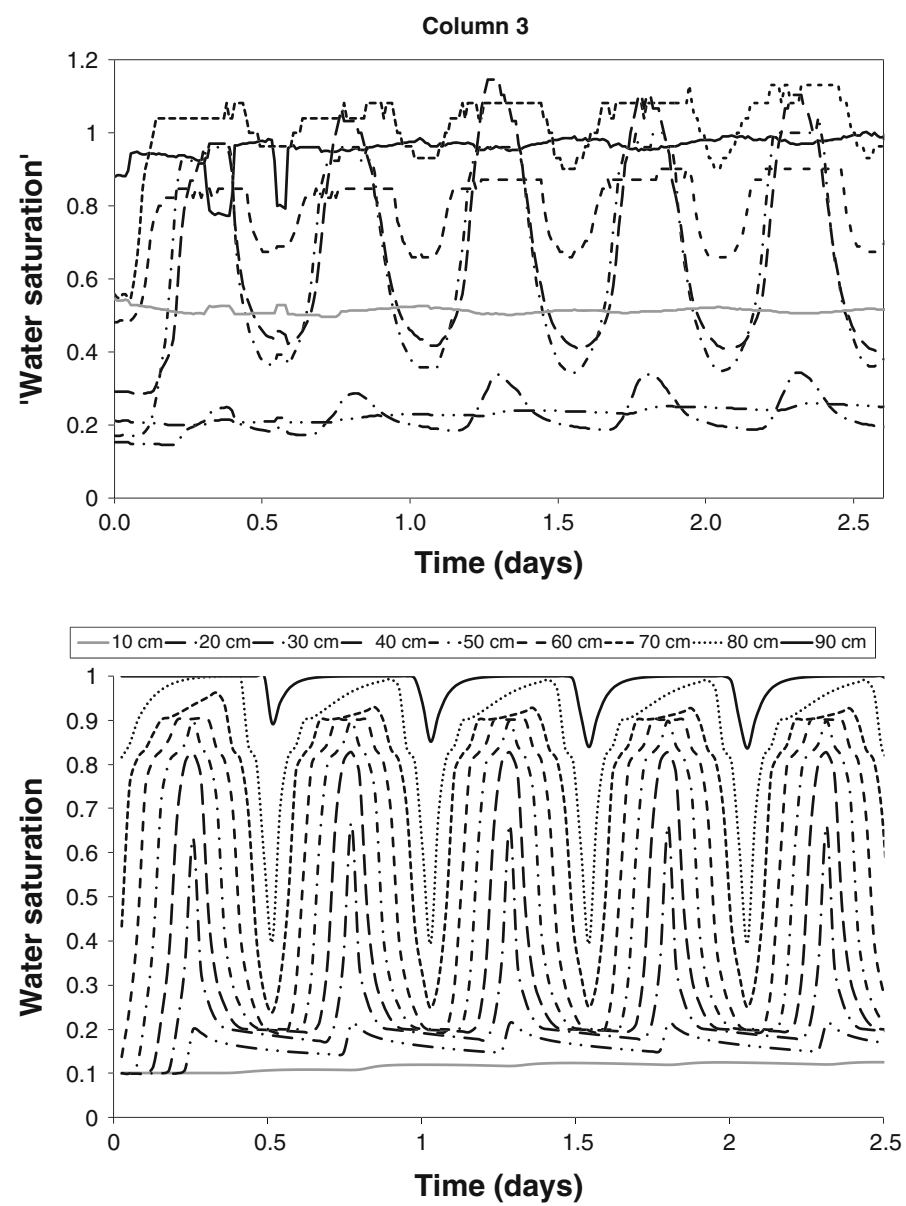

Fig. 6 Comparison of the change in water saturation seen with time in column 3 with that predicted by numerical simulation. The experimental water saturations were estimated from resistance. The saturation at the top of both experimental column and the simulation increases over time. The saturations elsewhere simply change periodically

speculate that this is because of different imbibition and drainage capillary curves resulting in their flows being more strongly influenced by gravity drainage.

The 'saturation' was seen to change with time at all levels in the experiments (although the magnitude of change was very small at the top and bottom of the columns) and in the simulations. It is interesting to note that saturation at $10 \mathrm{~cm}$ depth increased slowly over time in the simulations although this was not seen in the experiments. This effect is seen at $20 \mathrm{~cm}$ in the experiments, but is masked at $10 \mathrm{~cm}$ by the capillary end effect increasing the 'water saturation' near the top of the experimental columns.

Figure 7 shows the 'water saturation' profile with depth at maximum (high tide), decreasing water level, minimum free water level (low tide) and increasing free water level for each of the three columns after 25 days. These results (after 25 days) are typical of those seen through the period of tidal influence (from 0 to 90 days). There is very little change in 'water saturation' over 1 tidal cycle in column 1 . In column 2, the free water level increases by $10-15 \mathrm{~cm}$ from 

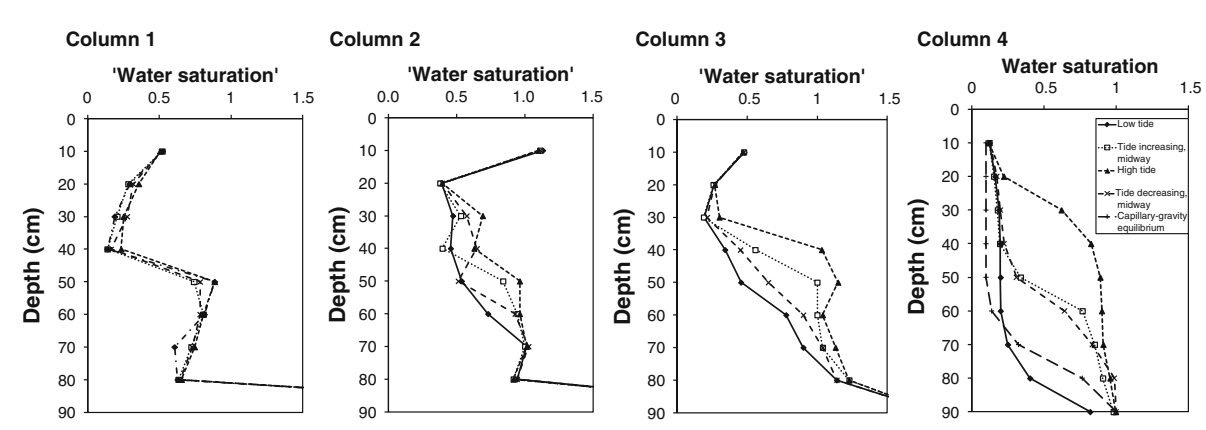

Fig. 7 Changes in water saturation with depth seen in each of the three columns and in numerical simulation at different states of the tide. The numerical simulation results have been averaged over 10 -cm intervals with a midpoint of the depth of each electrode pair so that they can be directly compared with the experimental observations. Note that 'Water saturation' goes from 0 to 1.5 to allow the resistivity change in the bottom pair of electrodes in each column to be seen. All graphs plotted for 25 days after the tidal oscillations began

low to high tide, while in column 3 the free water level changes by $15-20 \mathrm{~cm}$ from low to high tide. Water saturation changes are seen between 30 and $70 \mathrm{~cm}$ depth in columns 2 and 3 . As all the columns have very similar porosity (and hence permeability), it is possible that these differences between columns are due to different quantities entering and leaving each column. This is despite the fact that all three columns were fed by independent and identical tubes (in length and diameter) from the same water reservoir. An alternative explanation could be that resistances measured in these columns are less sensitive to changes in water saturation.

The simulation results, shown in Fig. $7 \mathrm{~d}$ are consistent with the experimental results seen in column 3 in terms of the magnitude and vertical distribution of the saturation changes. There are however subtle differences in the ordering of saturations between low and high tide. The 'water saturations' when the tide is increasing are generally higher than the 'water saturations' when the tide is decreasing. This is seen in both the simulation and the experiments and is presumably due to hysteresis.

Figure $7 \mathrm{~d}$ also shows the saturation profile seen at the start of the simulation, corresponding to that obtained by capillary-gravity equilibrium in the absence of a tidal change in water saturation. It is interesting that the simulations suggest that even the low tide water saturations are different from that expected from capillary-gravity equilibrium. The low tide saturations are lower in the vicinity of the free water level and higher further up the transition zone. These differences arise because there is not time for the water to drain completely under gravity from the upper parts of the column over the tidal period. This reduction in water saturation near the free water level and an increase above was also seen in the experiments of Stauffer and Kinzelbach (2001).

Figure 8 shows the period averaged 'saturation' seen at each depth in each column over 120 days. These saturations were obtained by taking a running average over one tidal period. There are some gaps in the data due to problems with the volume of data being logged. Close examination of these graphs shows that water saturation at some depths continue to change slowly over the entire 90 day period of tidal forcing, suggesting that the time constant for gravity drainage for this system is rather longer than the 3-21 day deduced by Iglauer and Muggeridge (2012). This is particularly apparent in column 3 at depths of 10,20, and $30 \mathrm{~cm}$ and in column 1 at a depth of $40 \mathrm{~cm}$.

All graphs in Fig. 8 show a rapid change in 'saturation' at intermediate depths after 90 days, when the stepper motor driving the periodic increase and decrease of the free water level was turned off. It can be seen that 'water saturation' decreases rapidly at depths of 

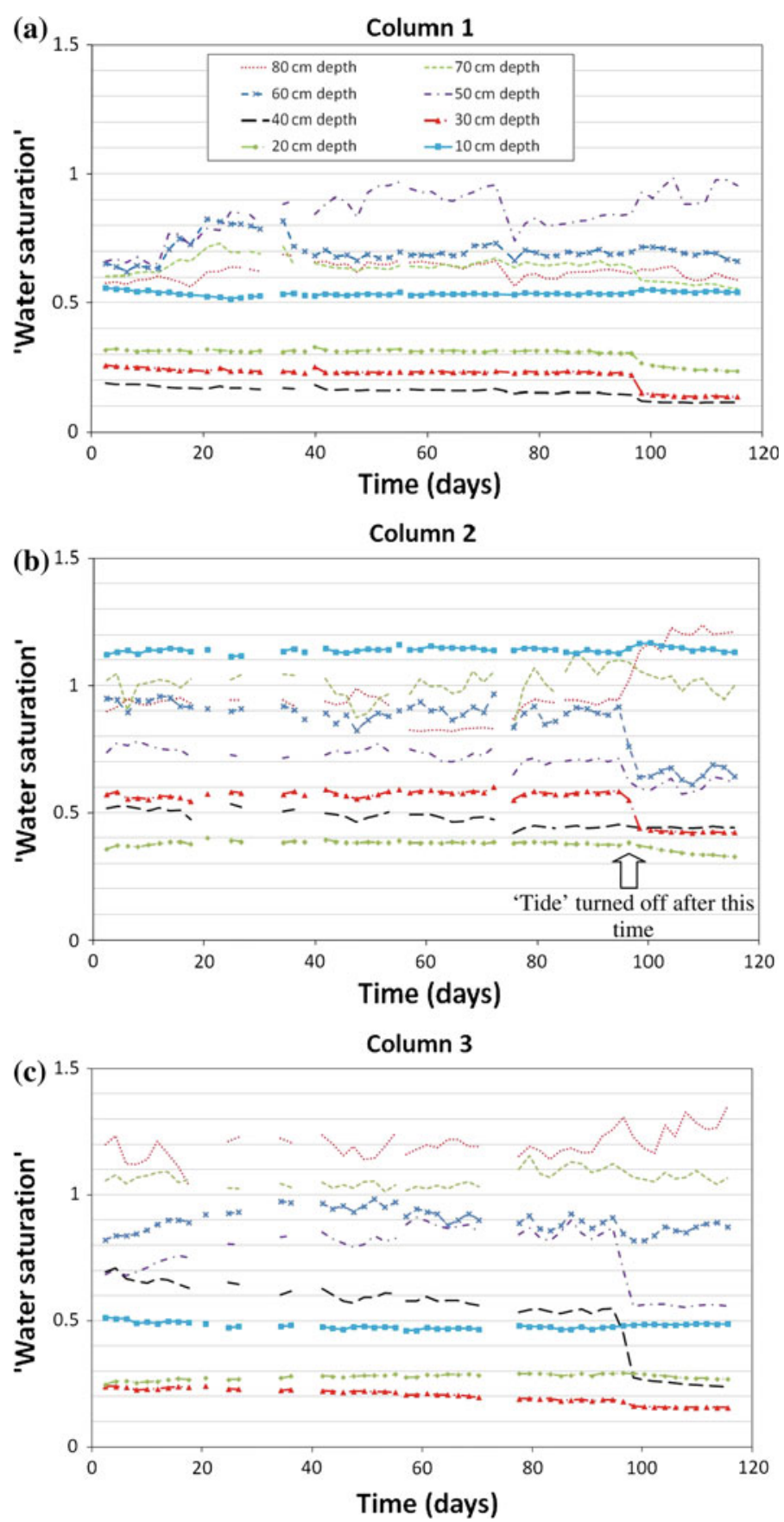

Fig. 8 Comparison of the 'water saturation' (resistance normalized to that measured at $100 \%$ water saturation) measured over 120 days. A running average over 1 tidal period has been applied to remove the periodic variations caused by the synthetic tide. Note that after 90 days, the synthetic tide was turned off. The gaps are areas where there were problems with data storage/acquisition 

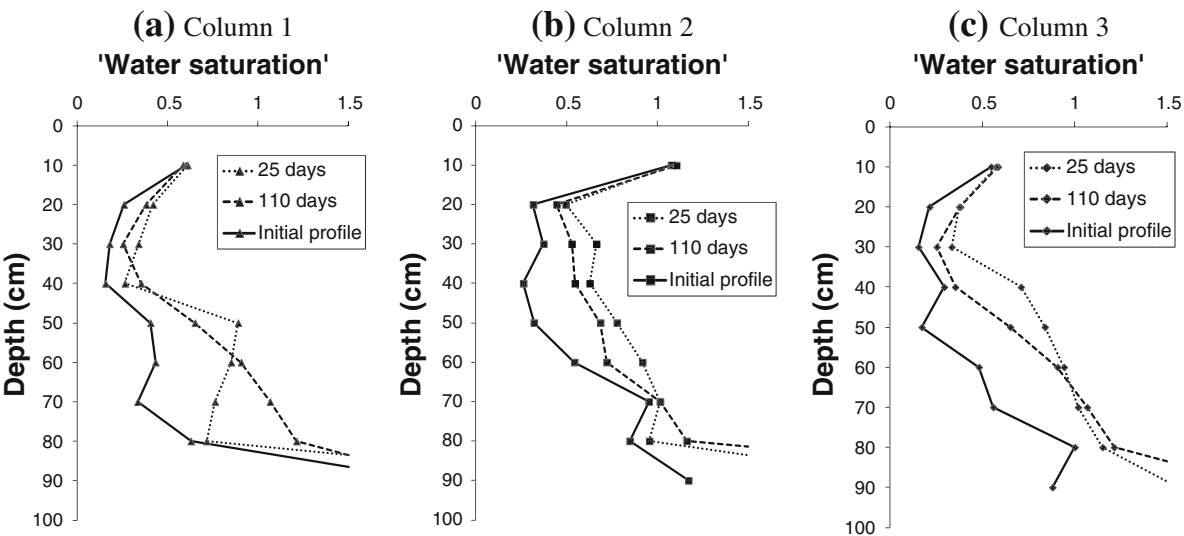

Fig. 9 Comparison of the water saturation profiles (averaged over 1 tidal period) versus depth seen in the three columns initially, after 25 days of tidal forcing and after 110 days (when tidal forcing had stopped for 20 days). The average water saturation when the columns undergo tidal forcing is higher than that seen under capillary-gravity equilibrium. Once the tidal forcing is removed, water drains under gravity so water saturation decreases between 20 and $60 \mathrm{~cm}$ depth and increases below $60 \mathrm{~cm}$

$20-40 \mathrm{~cm}$ at this time. In column 2 , there is also a corresponding increase in 'water saturation' at $80 \mathrm{~cm}$ at this time. This is consistent with the simulation results seen in Fig. $7 \mathrm{~d}$ in which the effect of the tidal variations in free water level was to increase the water saturation in the upper part of the transition zone above that which would be expected from the capillary gravity equilibrium using the drainage capillary pressure.

Figure 9 compares the period averaged 'water saturation' profiles versus depth seen in each of the three columns at 25 days (when the distribution was influenced by a tidally changing free water level) with that seen at 110 days (when the tidal changes have stopped) and the initial 'water saturation' profile. In all cases the water saturation seen between 40 and $80 \mathrm{~cm}$ depth for the tidally influenced cases are higher than those predicted or measured without tidal influence. This is also consistent with the results shown in Figs. 7d and 8.

\section{Discussion}

It is interesting to note that the average water saturation profile seen in Fig. 9, like the instantaneous profile seen at different points in the tidal period (Fig. 7) is not just the capillarygravity equilibrium curve shifted upwards. It has a different shape suggesting that the fluid distribution in transition zones (capillary fringes) with significant tidal influence cannot be predicted using drainage capillary pressure and capillary-gravity equilibrium. The change in shape will depend upon the height of the transition zone, the hysteresis in the capillary pressure and relative permeabilities, the time-scale for capillary imbibition versus gravity drainage and the magnitude of the tidal changes in pressure.

An observed increase in water saturation at intermediate depths when pressure was tidally influenced has also been seen by other workers. Ataie-Ashtiani et al. (2001) observed this in their numerical investigations into the height of the water table during tidally driven flow in an unconfined aquifer. It is also apparent in the experimental and numerical results of Wu and Zhuang (2010).

We would expect that the change in fluid distribution in the transition zone from that expected from capillary gravity equilibrium would be greatest where the time-scale for 
capillary imbibition of water is quicker than the tidal period and the time scale for gravity drainage is slow (longer than the tidal period). This is likely to be the case in low permeability oil reservoirs where the capillary pressure is high and the density difference between the water and the oil is low. The fluid distribution is also likely to be influenced by the hysteresis seen between drainage and imbibitions capillary pressure and relative permeability curves.

In oil reservoirs, there is often a change in wettability with height in the transition zone (e.g. Jackson et al. 2005) - higher up in the transition zone the rock becomes more mixed wet whereas our results were obtained for a strongly water-wet sand. This may reduce the influence of the tidal changes on the transition zone by reducing the imbibition of water into the upper parts of the transition zone or conversely, because the tides will have influenced the fluid distribution since the reservoir filled, the tidal influence may affect the change in wettability higher up in the transition zone.

We note that all the saturation changes at the different depths in the columns were in phase with the forcing and each other in both the simulation and the experiments. This is consistent with the analyses of Nielsen and Perrochet (2000) and Werner and Lockington (2003). These suggested that phase changes were only likely to occur for higher frequency pressure oscillations, i.e. with periods rather less than $6.5 \mathrm{~h}$. They predicted that the frequency response function for small amplitude oscillations in water table height would be given by:

$$
F(\omega)=\frac{1}{1+i\left(\phi_{d} \omega d / K\right)},
$$

where $\phi_{\mathrm{d}}$ is the effective dynamic porosity, $\omega$ is the angular forcing frequency, $d$ is the initial height of the water table and $K$ is the hydraulic conductivity. Thus phase changes would be greatest for higher forcing frequencies, higher porosities and low hydraulic conductivities. Although our packs had a relatively high porosity they also had a low forcing frequency $\left(1.42 \times 10^{-4} \mathrm{~Hz}\right)$ and a high hydraulic conductivity $\left(4.2 \times 10^{-4} \mathrm{~m} \mathrm{~s}^{-1}\right)$. From Eq. (3), this gives the value of the imaginary term to be $\sim 0.07$ suggesting that the phase change should be negligible, as was observed.

\section{Summary and Conclusions}

The influence of tidally influenced changes in free water level on the fluid distribution in the transition zone has been investigated experimentally and by using numerical simulation. The simulations used the same rock and fluid properties as measured from the experiments as far as possible. Both simulation and experiments gave qualitatively similar results in terms of the shape of periodically varying saturation at different depths as well as the average saturation profile with depths. No phase change in the water saturation versus time behaviour was noted with depth although the periodic function at intermediate depths was more like an asymmetric saw-tooth than a sinusoid. These observations were consistent with the analyses and observation seen in earlier works investigating the influence of waves and tides on the water table in coastal aquifers (Ataie-Ashtiani et al. 2001; Stauffer and Kinzelbach 2001; Wu and Zhuang 2010).

The investigation has shown that tidally induced changes in pressure may significantly alter the vertical saturation profile from that expected if capillary-gravity equilibrium is assumed. On average, a higher water saturation was observed above the mean free water level. Even at 'low tide', the vertical saturation profile differed from that expected from capillary-gravity equilibrium using the drainage capillary pressure curve. Just above the free water level, tidally influenced saturations were lower, while higher up the column, they were higher than those 
expected from capillary-gravity equilibrium. It seems likely that tidal influences may be one reason why the transition zone seen in oil reservoirs is not usually well described by capillary gravity equilibrium based on laboratory measurements of drainage capillary pressure.

Further investigation is needed to confirm this inference as the transition zone investigated in these experiments was constrained by the design of the experiments to be only slightly bigger than the tidal variations in depth of the free water level. As a result, the flow was more dominated by gravity rather than capillary pressure. In a reservoir, the transition zone would be rather bigger than the tidal variations in free water level, i.e. flow would be more dominated by capillary pressure. In addition the porous media in our study were unconsolidated sand packs, and thus had a higher compressibility than typical rock found in oil reservoirs. This will have tended to reduce the effect of tidal changes on water saturation above the free water level. Our experimental measurements used resistance to observe changes in water saturation. The resistance data were also influenced by changes in porosity resulting from the high compressibility of the sand packs, resulting in observations of water saturation that were apparently greater than 1 . Any further experimental investigations should include quantitative calibration of resistance versus saturation through direct measurement on a small, control sand pack. In addition, care should be taking to ensure the sand is densely packed and thus reduce the likelihood of grain movement during flow.

Acknowledgments Graham Nash is thanked for his help in manufacturing the columns used in these experiments. Olaware Kolawole is thanked for her help in performing some preliminary data analysis and numerical simulations. The work was funded by the UK Engineering and Physical Sciences Research Council (Grant No: EP/D075424/1).

Open Access This article is distributed under the terms of the Creative Commons Attribution License which permits any use, distribution, and reproduction in any medium, provided the original author(s) and the source are credited.

\section{References}

Aggelopoulos, C., Klepetsanis, P., Theodoropoulou, M.A., Pomoni, K., Tsakiroglou, C.D.: Large-scale effects on resistivity index of porous media. J. Contam. Hydrol. 77(4), 299-323 (2005)

Archie, G.E.: The electrical resistivity $\log$ as an aid in determining some reservoir characteristics. Trans. AIME 146, 54-67 (1942)

Ataie-Ashtiani, B., Volker, R.E., Lockington, D.A.: Tidal effects on groundwater dynamics in unconfined aquifers. Hydrol. Process. 15, 655-669 (2001). doi:10.1002/hyp.183

Bredehoeft, J.D.: Response of well-aquifer systems to Earth tides. J. Geophys. Res. 72(12), 3075-3087 (1967)

Bunn, M.I., Jones, J.P., Endres, A.L., Rudolf, D.L.: Effects of hydraulic conductivity heterogeneity on vadose zone response to pumping in an unconfined aquifer. J. Hydrol. 387(1-2), 90-104 (2010). doi:10.1016/j. jhydrol.2010.03.036

Butikov, E.I.: A dynamical picture of the oceanic tides. Am. J. Phys. 70, 1001-1011 (2002)

Camps-Roach, G., O'Carroll, D.M., Newson, T.A., Sakaki, T., Illangasekare, T.H.: Experimental investigation of dynamic effects in capillary pressure: grain size dependency and upscaling. Water Resour. Res. 46, 1-13 (2010)

Cartwright, N., Nielsen, P., Perrochet, P.: Influence of capillarity on a simple harmonic oscillating water table: sand column experiments and modeling. Water Resour. Res. 41, (2005). doi:10.1029/2005WR004023.

Cartwright N., Nielsen P., Perrochet P.: Behavior of a shallow water table under periodic flow conditions. Water Resour. Res. 45 (2009). doi:10.1029/2008WR007306.

Chang, E., Firoozabadi, A.: Gravitational potential variations of the Sun and Moon for the estimation of reservoir compressibility. SPE J. 5, 456-465 (2000)

Dake, L.P.: Fundam. Reserv. Eng. Elsevier, Amsterdam (1983)

Dean, G.A., Hardy, R., Eltvik, P.: Monitoring compaction and compressibility changes in offshore chalk reservoirs. SPE Form. Eval. 9(1), 73-76 (1994) 
Di Donato, G., Tavassoli, Z., Blunt, M.J.: Analytical and numerical analysis of oil recovery by gravity drainage. J. Pet. Sci. Eng. 54, 55-69 (2006)

Dullien, F.A.L.: Porous Media: Fluid Transport and Pore Structure, 2nd edn. Academic Press, San Diego (1992)

Fanchi, J.R., Chistiansen, R.L., Heymons, M.J.: Estimating oil reserves of fields with oil/water transition zones. SPE Reserv. Eval. Eng. 5(4), 311-316 (2002)

Hanson, J.M., Owen, L.B.: Fracture orientation analysis by the solid earth tidal strain method. In: SPE 11070 presented at the 57th SPE Annual Fall Technical Conference and Exhibition held in New Orleans, Louisiana (1982).

Huang, D.D., Honarpour, M.M. (1996) Capillary end effects in coreflood calculations. In: SCA Conference Paper Number 9634, Presented at the International Symposium of the Society of Core Analysts held in Montpellier, France.

Ivanov, D.A., Araujo, M.: Dynamics of two-phase immiscible pulsed flow. In: SPE 99678 Presented at the SPE/DOE Symposium on Improved Oil Recovery held in Tulsa, Oklahoma (2006). doi:10.2118/99678-MS.

Iglauer, S., Muggeridge, A.H.: Time dependence of free fall gravity drainage in unconsolidated sand. J. Porous Med. 15(8), 721-733 (2012)

Jackson, P.D., Taylor-Smith, D., Stanford, P.N.: Resistivity-porosity-particle shape relationships for marine sands. Geophysics 43(6), 1250-1268 (1978)

Jackson, M.D., Valvatne, P.H., Blunt, M.J.: Prediction of wettability variation within an oil/water transition zone and its impact on production. SPE J. 10(2), 185-195 (2005)

Killough, J.E.: Reservoir simulation with history-dependent saturation functions. Trans. AIME 261, 37-48 (1976)

Kuruana, A.K.: Influence of tidal phenomenon on interpretation of pressure build up and pulse test. APEA J. 16(1), 99-105 (1976)

Langaas, K., Nilsen, K.I., Skjaeveland, S.M.: Tidal pressure response and surveillance of water encroachment. SPE Reserv. Eval. Eng. 9(4), 335-344 (2006)

Lehmann, P., Stauffer, F., Hinz, C., Dury, O., Fluhler, H.: Effect of hysteresis on water flow in a sand column with a fluctuating capillary fringe. J. Contam. Hydrol. 33(1-2), 81-100 (1998)

Li, L., Barry, D.A., Stagnitti, F., Parlange, J.-Y.: Groundwater waves in a coastal aquifer: a new governing equation including vertical effects and capillarity. Water Resour. Res. 36(2), 411-420 (2000)

Lide, D.R.: CRC Handbook of Chemistry \& Physics, 87th edn. CRC Press (2007)

Masmaleh, S.K., Abu Shiekah, I., Jing, X.D.: Improved characterization and modeling of capillary transition zones in carbonate reservoirs. SPE Reserv. Eval. Eng. 10(2), 191-204 (2007)

Neeper, D.A.: A model of oscillatory transport in granular soils with application to barometric pumping and earth tides. J. Contam. Hydrol. 48(3-4), 237-252 (2001)

Nielsen, P., Perrochet, P.: Watertable dynamics under capillary fringes: experiments and modeling. Adv. Water Resour. 23, 503-515 (2000)

O’Carroll, D.M., Abriola, L.M., Polityka, C.A., Bradford, S.A., Demond, A.H.: Prediction of two-phase capillary pressure-saturation relationships in fractional wettability systems. J. Contam. Hydrol. 77, 247270 (2005)

Parker, A.R., Rudd, J.M.: Understanding and modelling water free production in transition zones: a case study. In: SPE 59412. Proceedings of the 2000 SPE Asia Pacific Conference on Integrated Modelling for Asset Management, Yokohama, Japan (2000)

Sahni, A.: Measurements of three phase relative permeability during gravity drainage using CT scanning. $\mathrm{Ph}$. D. Thesis, Stanford University (1998)

Serway, R.A.: Principles of Physics. Saunders College Publishing, London (1998)

Soner, S., Amabeoku, M., Kissami, M.: Formation resistivity response to loading and unloading confining pressure. J. Pet. Sci. Eng. 16, 169-179 (1996)

Stauffer, F., Kinzelbach, W.: Cyclic hysteretic flow in porous medium column: model, experiment and simulations. J. Hydrol. 240, 264-275 (2001)

Wakeham, R.J., Vince, A.: Kinetics of gravity drainage from porous media. Chem. Eng. Res. Des. 64, 94-103 (1986)

Werner, A.D., Lockington, D.A.: Influence of hysteresis on tidal capillary fringe dynamics in a well-sorted sand. Adv. Water Resour. 26, 1199-1204 (2003)

Wu, L.H., Zhuang, S.Y.: Experimental investigation of effect of tide on coastal ground water table. J. Hydrodyn. 22(1), 66-70 (2010). doi:10.1016/S1001-6058(09)60029-9

Wyllie, M.R.J., Rose, W.D.: Some theoretical considerations related to the quantitative evaluation on the physical characteristics of reservoir rock from electrical log data. Trans. AIME 189, 105-118 (1950) 